\title{
ISO^ observations of four active galaxies
}

\author{
M. Dennefeld ${ }^{1, \star \star}$, T. Boller ${ }^{2}$, D. Rigopoulou ${ }^{2}$, and H. W. W. Spoon ${ }^{3}$
}

\author{
1 Institut d'Astrophysique de Paris, 98bis Bd Arago, 75014 Paris, France \\ 2 Max-Planck Institut for Extraterrestrial Physics, 85740 Garching, Germany \\ 3 Kapteyn Astronomical Institute, 9700 AV Groningen, The Netherlands
}

Received 23 December 2002 / Accepted 23 April 2003

\begin{abstract}
We present ISO PHOT-S spectra of four galaxies known or suspected to host a central AGN. Two of them are selected, among several others, from the initial Iras/Rosat sample of Boller et al. (1992) because of their substantial X-ray emission, while no obvious Seyfert features was present in their optical spectra: IRAS 14201+2956 and IRAS 21582+1018. The latter, also known as Mrk 520, was bright enough to also allow SWS observations around selected neon lines, to establish its excitation. While both PHOT-S spectra are characteristic of starburst-dominated galaxies, the neon line ratios in IRAS $21582+1018$ indicate the presence of a hard excitation source. Complementary optical spectra, both at low and high spectral resolution, show only a weak, broad component around $\mathrm{H}_{\alpha}$, establishing the presence of a central AGN which may not be detected in standard, classification spectra. Both objects are now classified as Sey 1.9 galaxies. These results show therefore that, although IR observations were expected to be able to pierce through the dusty central regions to reveal the presence of an active nucleus, the result may be ambiguous: the broad band IR energy distribution can still be dominated by starburts located in a wider circumnuclear region, and the AGN appear only in specific observations (high-excitation lines in the IR, or high-resolution optical spectra). As a complement, two other galaxies from the same initial sample were also observed with PHOT-S: the Narrow Line Seyfert galaxies (NLS1) Mrk 359 and Mrk 1388. NLS1s appear in high proportion in the Rosat/Iras sample, and in soft X-ray samples in general, and their Balmer line-widths are sometimes comparable to those of interacting, star-forming galaxies. Their ISO spectra however do not reveal the typical, strong PAH features found in the starburst galaxies and are more like those of standard Seyferts. All these observations therefore indicate that the key element is the presence or absence of a circumnuclear starburst region which, if strong enough, may completely hide the presence of a central AGN in the IR spectral energy distribution. The dust obscuration however needs to be patchy rather than complete to explain the detection of the high-excitation lines and Balmer wings in some cases. Only high-energy observations can then establish the strength of the central AGN and the amount of extinction with certainty.
\end{abstract}

Key words. galaxies: active - galaxies: starburst - infrared: galaxies - X-rays: galaxies

\section{Introduction}

The comparison between the ROSAT All Sky Survey (RASS) and the IRAS Point Source Catalog revealed many galaxies with X-ray luminosity in the range $10^{42}-10^{43} \mathrm{erg} \mathrm{s}^{-1}$, which had not been previously classified as Active Galactic Nuclei (AGN) (Boller et al. 1992; Boller et al. 1998; hereafter B92 and B98). For many of them, this was simply due to the lack of optical spectroscopy, but for some others, the existing, low-dispersion spectra did not reveal clear characteristics of a Seyfert type nucleus. These cases opened the possibility that AGN may exist in many galaxies without being detected by standard spectroscopy (Boller et al. 1993) and deserved further studies. As the selection process of this sample included detection by IRAS, it was also clear that obscuration

Send offprint requests to: M. Dennefeld, e-mail: dennefel@iap. fr

* An ESA project with instruments funded by ESA member states.

$\star \star$ Visiting astronomer, Observatoire de Haute-Provence (OHP), CNRS, France. by dust was an important factor, as shown by the results obtained in various IRAS galaxies samples (e.g. Heckman et al. 1987; Veilleux et al. 1995). In particular, the controversy about the nature of the dominant energy source, starburst or AGN, in the high-luminosity IRAS galaxies (Sanders \& Mirabel 1996, and references therein) was illustrative of the questions raised by IR-selected objects.

A systematic optical spectroscopic follow-up of the B92 sample was therefore undertaken, the results of which will be presented elsewhere (Dennefeld et al., in preparation). Independant observations were also published by Moran et al. (1996). But at the same time, the availability of the Infrared Space Observatory (ISO) of the European Space Agency (Kessler et al. 1996) allowed specific mid-infrared spectroscopy to be envisaged for those objects with high X-ray luminosity and no obvious AGN signature in the visible, with the hope that the smaller extinction expected at these IR wavelengths would allow a better determination of the nature of the nuclear source. Observations of a large number 

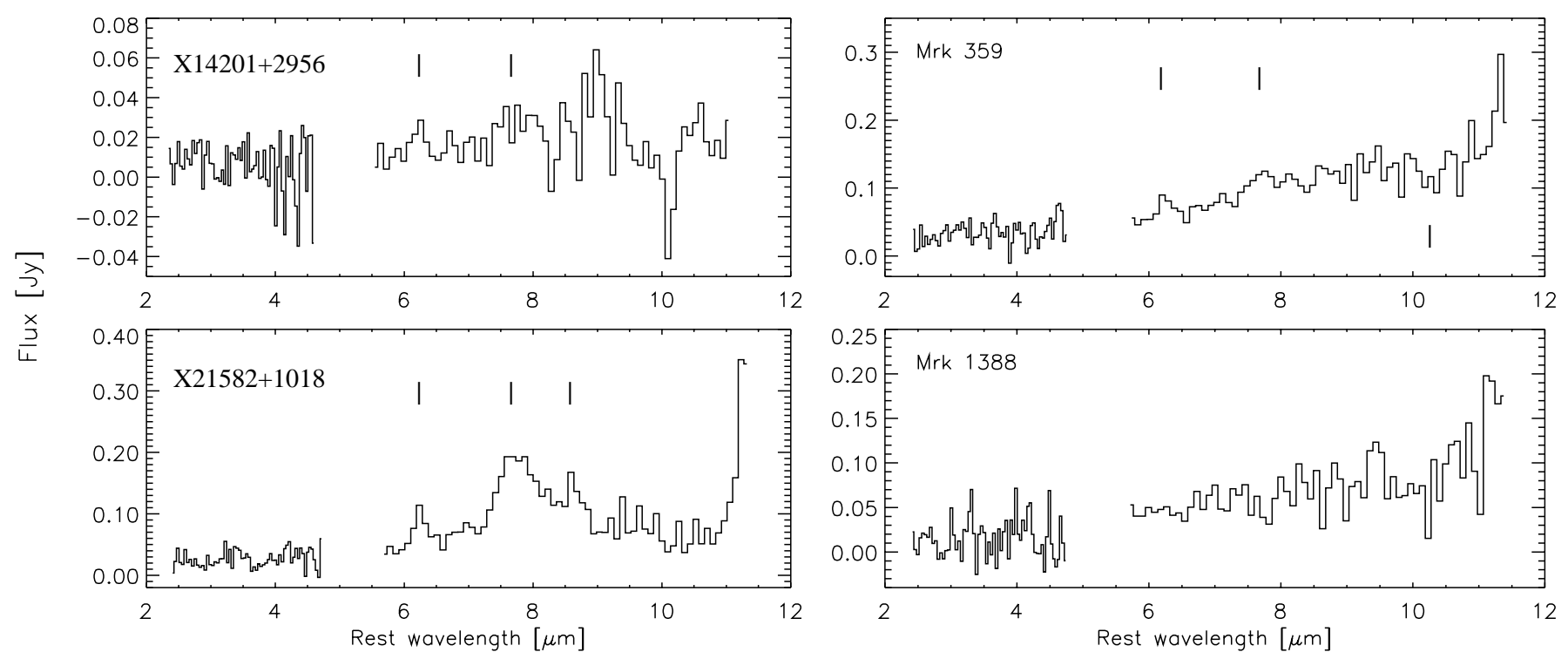

Fig. 1. PHOT-S spectra of the four galaxies. On the left, the two "starburst" galaxies, IRAS 14201+2956 and IRAS 21582+1018 (alias Mrk 520); on the right, the two NLS1 objects, Mrk 359 and Mrk 1388. Tickmarks indicate the detected lines.

of reference objects, both starburst and Seyfert galaxies, in the ISO core-program, would provide the necessary reference for this identification: indeed, results by Genzel et al. (1998) show that both fine structure lines observed with the Short Wavelength Spectrometer (SWS) and polycyclic aromatic hydrocarbon (PAH) features observed at lower spectral resolution with the ISOPHOT-S are excellent diagnostics to distinguish starburst and AGN energy sources.

The allocated observing time and the effective ISO sensitivity allowed us to observe only a few, bright objects, among the many initially selected. Two of them belong to the subclass of X-rays bright objects without previous Seyfert classification from the B92 sample: IRAS 14201+2956 and IRAS $21582+1018$, the second one also being known as Mrk 520 (Markarian \& Lipovetskii 1974). To those were added two other, bright, Narrow Line Seyfert galaxies (NLS1): Mrk 359 (Markarian \& Lipovetskii 1971) and Mrk 1388 (Markarian et al. 1980). This class of objects was not well represented in the Genzel et al. (1998) core-program, but appeared in large proportions in the B92 sample. The Balmer linewidths of NLS1 are much smaller than in standard Seyfert 1s, and are sometimes comparable to those of interacting, starforming galaxies, raising the question of a possible link between the peculiarities of the NLS1 and the properties of dusty starbursts/AGNs.

The basic parameters of these four objects are presented in Table 1. To facilitate the separation between the two types of objects, we will call the two NLS1s by their Markarian names, and refer to the two other objects by their IRAS name.

In this paper, we report the results of the ISO observations of these four targets, together with those of complementary optical observations. We will assume $H_{0}=50 \mathrm{~km} \mathrm{~s}^{-1} \mathrm{Mpc}^{-1}$, but the exact value has little influence on our conclusions. Energies are quoted in the source rest frame. The ISO observations are presented in Sect. 2, the other data in Sect. 3 (X-rays, radio, optical), and the results are discussed in the last section.

\section{ISO observations and data reduction}

\subsection{The PHOT-S observations}

All four objects were observed in 1996-97 with the ISOPHOT-S spectrophotometer (Lemke et al. 1996) in rectangular chopped mode, with on-source integration times of $512 \mathrm{~s}$. The total spectral range is covered simultaneously by two linear arrays, from 2.47 to $4.87 \mu \mathrm{m}$ and from 5.84 to $11.62 \mu \mathrm{m}$ respectively, with a common entrance aperture of $24^{\prime \prime} \times 24^{\prime \prime}$. The ISOPHOT-S data were reduced using the PIA version 8.1: the main steps of reduction can be found in Rigopoulou et al. (1999). But it is worth mentioning here that, with this version, the short-wavelength range is also satisfactorily processed and no additionnal flux correction factor is necessary. The resulting spectra are shown in Fig. 1 and some measured values can be found in Table 2 (Cols. 4 to 8 ).

\subsection{SWS observations}

The brightest source, Mrk $520=$ IRAS 21582+1018, was also observed in late 1996 with SWS, in selected wavelength ranges around the Neon lines ([Ne II] at 12.8, [Ne V] at 14.3 and [Ne III] at $15.5 \mu \mathrm{m}$ rest wavelength respectively) in the high resolution SWS AOT6 mode. In this configuration, the entrance slit is $14^{\prime \prime} \times 20^{\prime \prime}$, slightly smaller than the aperture used in PHOT observations. The data were reduced using the SWS Interactive Analysis (IA) data reduction software package using standard ISO pipeline data routines and the corresponding calibration files (version 9.5). We have also used some more sophisticated software tools to improve dark current subtraction and flat fielding. The final spectra were re-binned to resolutions between 1200 and 1500 . The resulting lines together with Gaussian line fits (used to determine line fluxes) are shown in Fig. 2. 
Table 1. Basic properties of the four observed sources. IRAS $21582+1018$ is also known as Mrk 520 .

\begin{tabular}{lcccccc}
\hline \hline Source & $\alpha_{2000}$ & $\delta_{2000}$ & $\begin{array}{c}V_{\odot} \\
\left(\mathrm{km} \mathrm{s}^{-1}\right)\end{array}$ & $\begin{array}{c}L_{I R} \\
\left(10^{10} L_{\odot}\right)\end{array}$ & $\begin{array}{c}L_{X}(0.5-2 \mathrm{keV}) \\
\left(10^{42} \mathrm{ergs} \mathrm{s}^{-1}\right)\end{array}$ & $\begin{array}{c}\text { IRAS } \\
(25 / 60 \mu \mathrm{m} \text { flux ratio })\end{array}$ \\
\hline Mrk 359 & $01^{\mathrm{h}} 27^{\mathrm{m}} 32^{\mathrm{s}} .3$ & $19^{\circ} 10^{\prime} 39^{\prime \prime}$ & 5100 & 3.28 & 34.1 & 0.39 \\
Mrk 1388 & $14^{\mathrm{h}} 50^{\mathrm{m}} 37^{\mathrm{s}} .8$ & $22^{\circ} 44^{\prime} 04^{\prime \prime}$ & 6350 & 2.34 & 2.3 & 1.33 \\
IRAS 14201+2956 & $14^{\mathrm{h}} 22^{\mathrm{m}} 20^{\mathrm{s}} .2$ & $29^{\circ} 42^{\prime} 55^{\prime \prime}$ & 15700 & 15.8 & 13.7 & 0.21 \\
IRAS 21582+1018 & $22^{\mathrm{h}} 00^{\mathrm{m}} 41^{\mathrm{s}} .4$ & $10^{\circ} 33^{\prime} 08^{\prime \prime}$ & 8200 & 23.1 & 5.7 & 0.12 \\
\hline
\end{tabular}

Table 2. Best-fit parameters and results. The two first columns refer to the X-rays data, the last ones to the ISO data.

\begin{tabular}{lccccccc}
\hline \hline Source & $\begin{array}{c}N_{\mathrm{H}} \\
\left(10^{20} \mathrm{~cm}^{-2}\right)\end{array}$ & $\Gamma$ & $\begin{array}{c}5.9 \mu \mathrm{m} \text { Cont. } \\
(\mathrm{mJy})\end{array}$ & $\begin{array}{c}7.7 \mu \mathrm{m} \text { Cont. } \\
(\mathrm{mJy})\end{array}$ & $\begin{array}{c}7.7 \mu \mathrm{m} \text { PAH flux } \\
\left(10^{-20} \mathrm{~W} \mathrm{~cm}^{-2}\right)\end{array}$ & $\begin{array}{c}7.7 \mu \mathrm{m} \\
\mathrm{L} / \mathrm{C} \text { ratio }\end{array}$ & $\begin{array}{c}6.2 \mu \mathrm{m} \text { PAH flux } \\
\left(10^{-20} \mathrm{~W} \mathrm{~cm}^{-2}\right)\end{array}$ \\
\hline Mrk 359 & $5.7(4.8) \dagger$ & $2.7 \pm 0.4$ & $53.1 \pm 3.3$ & $81.2 \pm 7.4$ & $12.2 \pm 1.7$ & 0.22 & $5.0 \pm 1.1$ \\
Mrk 1388 & $9.6(2.8)$ & $1.8 \pm 2.2$ & $43.0 \pm 3.4$ & $53.7 \pm 7.6$ & $\leq 5.78$ & & $\leq 3.5$ \\
IRAS 14201 & $1.7(1.3)$ & $1.7 \pm 0.8$ & $10.4 \pm 2.1$ & $19.2 \pm 5.7$ & $\leq 3.74$ & & $2.3 \pm 0.9$ \\
IRAS 21582 & $20(5.4)$ & $3.0 \pm 2.5$ & $40.2 \pm 2.9$ & $55.8 \pm 6.7$ & $47.4 \pm 1.7$ & 1.42 & $12.7 \pm 1.2$ \\
\hline
\end{tabular}

${ }^{\dagger}$ Galactic value in parentheses.

\section{Additional data at other wavelengthes}

\subsection{X-rays data}

The four objects were selected from a cross-correlation (B92) between the Iras data and the Rosat All-Sky Survey; the latter is the main source for X-rays data. For each of the objects, a power law fit was done over the $0.1-2.4 \mathrm{keV}$ spectral range, with free photon index $\Gamma$ and hydrogen column density $N_{\mathrm{H}}$. The results for $N_{\mathrm{H}}$ and $\Gamma$ are given in the first two columns of Table 2. The value for the Galactic foreground absorbing column density (Dickey \& Lockman 1990, and references therein) is given in parentheses after the value obtained from the best fit. The absorption corrected flux is then used to derive the X-rays luminosity given in Table 1.

For Mrk 1388 and IRAS $21582+1018$ the spectral fit is poorly constrained. An alternative flux determination can then be done by fitting a power law with fixed spectral index (2.3 used here) and only the Galactic foreground absorbing column density. The resulting flux, 1.0 and $3.3 \times 10^{42} \mathrm{ergs} \mathrm{s}^{-1}$ respectively, is a factor of two lower than the result from the spectral fit, consistent with the lower absorbing column density used, and gives an estimate of the uncertainties. For IRAS $21582+1018$ the optical galaxy is offset from the centroid of the X-ray position but lies within the contours; no other optical object is visible within these contours down to $B=23$ at least, and the galaxy is the only candidate, although the contribution from a neutron star cannot be formally excluded. IRAS $14201+2956$ is in fact a pair of galaxies, but the X-ray source is clearly related to one of the components only (the SW one), to which the ISO and optical spectra refer also. No bridge is seen between those two galaxies on deep optical images obtained at OHP.

No indication for variability is apparent from the Rosat data, except for IRAS $14201+2956$. This is also the only object for which other X-ray data are available: it has been observed in the Einstein extended medium-sensitivity survey
(Gioia et al. 1990) with a flux of $(7.16 \pm 0.83) \times$ $10^{-13} \mathrm{ergs} \mathrm{cm}^{-2} \mathrm{~s}^{-1}$ in the $0.3-3.5 \mathrm{keV}$ range. The Rosat flux of $1.15 \times 10^{-12} \mathrm{ergs} \mathrm{cm}^{-2} \mathrm{~s}^{-1}$, in the smaller spectral range of $0.1-$ $2.4 \mathrm{keV}$, is indeed higher, but this could be due to the soft-X excess only. We have used the power law fit done over the Rosat spectral range to evaluate the flux in the Einstein band $(0.3-$ $3.5 \mathrm{keV}$ ) and found a value of $5.9 \times 10^{-13} \mathrm{ergs} \mathrm{cm}^{-2} \mathrm{~s}^{-1}$. The difference with the original Einstein observations appears to be marginally significant and could be indicative of time variations also.

No hard X-ray data are available for the moment for any of those objects, making difficult the analysis of the energy source from the X-ray point of view alone.

\subsection{Optical observations}

Complementary spectra were obtained over several observing runs at Haute-Provence Observatory (OHP) with the $1.93 \mathrm{~m}$ telescope, both at low and high dispersion. The first spectra, at $7 \AA$ per pixel, were obtained for IRAS $14201+2956$ (Fig. 3) and IRAS 21582+1018 in May 1992, and did not show any clear evidence of a Seyfert nature. In the spectrum of IRAS $21582+1018$ some line ratios $\left([\mathrm{NII}] / \mathrm{H}_{\alpha}\right.$ or $[\mathrm{SII}] / \mathrm{H}_{\alpha}$ ) show higher values than in standard HII regions, but not high enough to firmly establish the presence of an AGN (they could as well be produced by supernova-remnants). This surprising result in view of the strong X-ray emission called for additional efforts. Confirmation spectra at $7 \AA$ per pixel were therefore obtained again, as well as spectra at a better resolution of $1.8 \AA$ per pixel (in either the $\mathrm{H}_{\alpha}$ or the $\mathrm{H}_{\beta}$ spectral region). For IRAS 21582+1018 this was done in September 1996: Fig. 4 shows the low-dispersion spectrum, with better signal to noise than the 1992 one, while the high-dispersion one is presented in Fig. 5. In the mean time, Moran et al. (1996) published also a spectrum of this object, classifying it as a Sey 1.9. For IRAS $14201+2956$ a second low dispersion spectrum was secured in May 1995, but only the $\mathrm{H}_{\alpha}$ region could be observed 

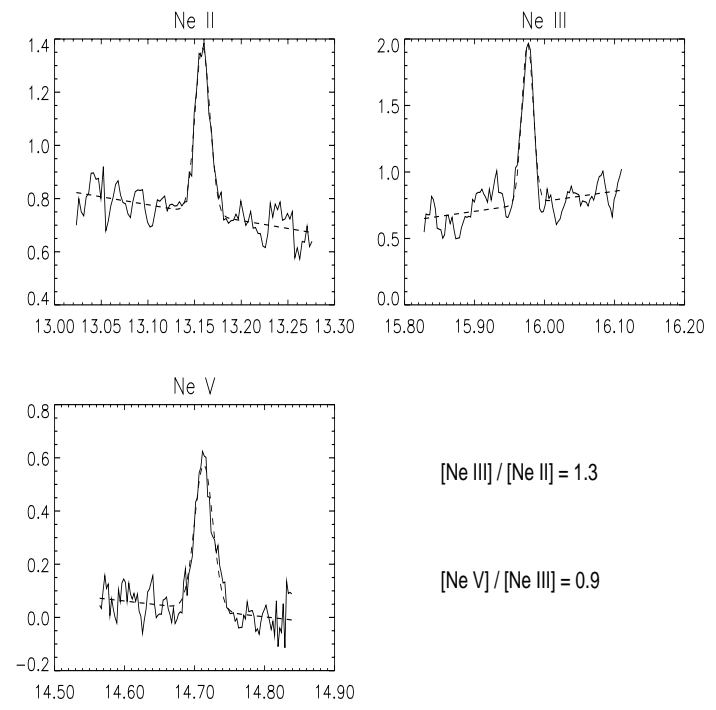

$[\mathrm{Ne} I I I] /[\mathrm{Ne} I I]=1.3$

$[\mathrm{Ne} \mathrm{V}] /[\mathrm{Ne} \mathrm{III]}]=0.9$

Fig. 2. Individual Neon lines detected in IRAS $21582+1018$.

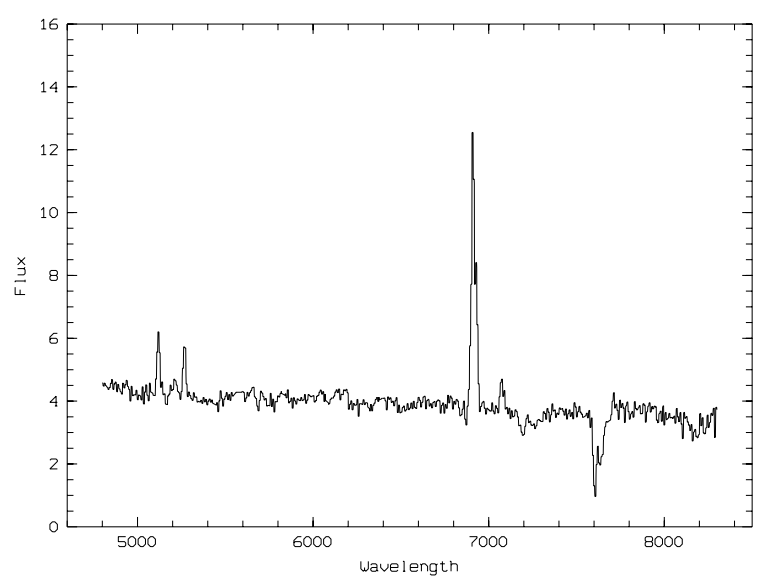

Fig. 3. Low dispersion spectrum of IRAS 14201+2956 obtained in May 92. Note the absence of conspicuous Seyfert features.

at higher resolution during this run. Both the $\mathrm{H}_{\alpha}$ and the $\mathrm{H}_{\beta}$ region were re-observed during another run in May 1998 and the $\mathrm{H}_{\alpha}$ part is presented in Fig. 6 . The dashed lines in these figures represent the result of multi-component analysis, which will be discussed in Sect. 4.2. Low dispersion spectra were obtained for the known Seyferts Mrk 359 (January 1996; Fig. 7) and Mrk 1388 (June 1999; Fig. 8), to check for eventual variability, but no conspicuous changes were noted. All spectra were reduced in the standard way and calibrated in flux with the help of standard stars observed with the same setting during the same runs. The relative fluxes are estimated to be accurate within $10 \%$ (except at the very blue end were the atmospheric extinction is not well calibrated). Absolute fluxes are not given systematically, as the observing conditions were not perfect in each of the runs. Small variations of the total magnitude of an object from one run to the next can therefore not be estimated here.

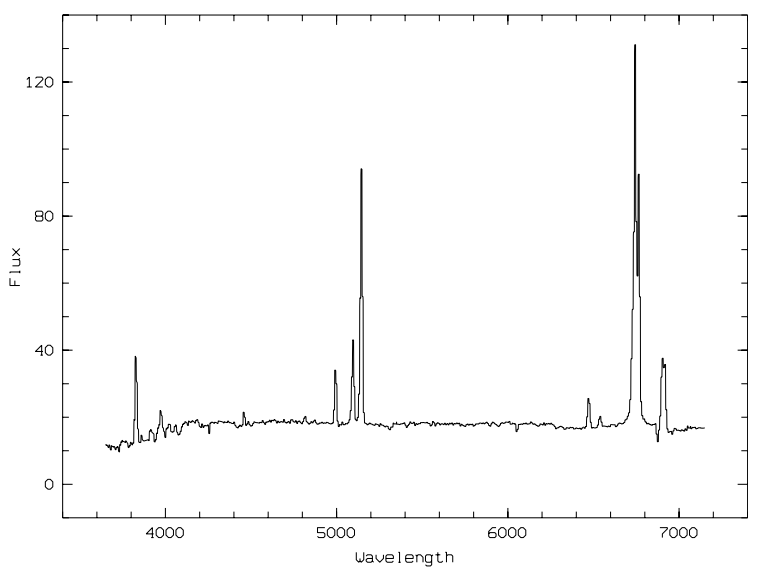

Fig. 4. Low dispersion spectrum of IRAS $21582+1018$ obtained in Sep. 96. No broad Aline features are apparent, but line ratios are close to the borderline between AGN and HII type galaxies.

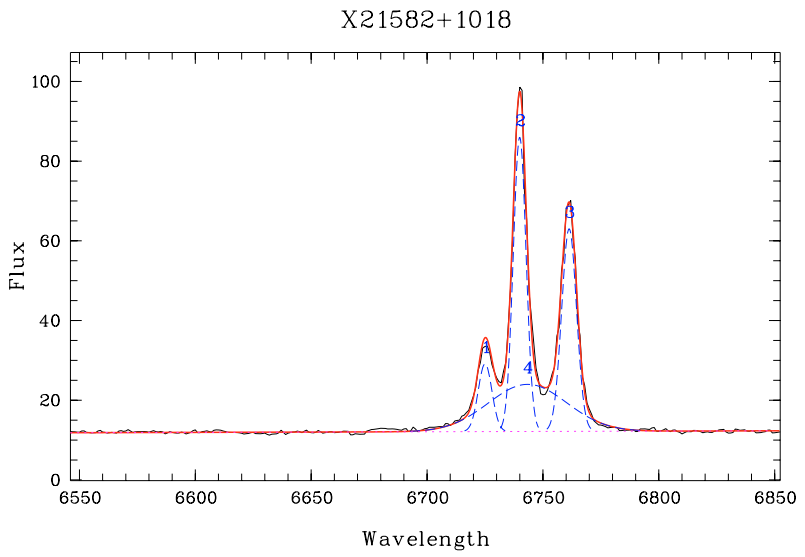

Fig. 5. IRAS $21582+1018$ high dispersion spectrum (Sep. 96) in the $\mathrm{H}_{\alpha}$ region. The presence of a broad $\mathrm{H}_{\alpha}$ component can only be established by multi-component fiting (dashed lines).

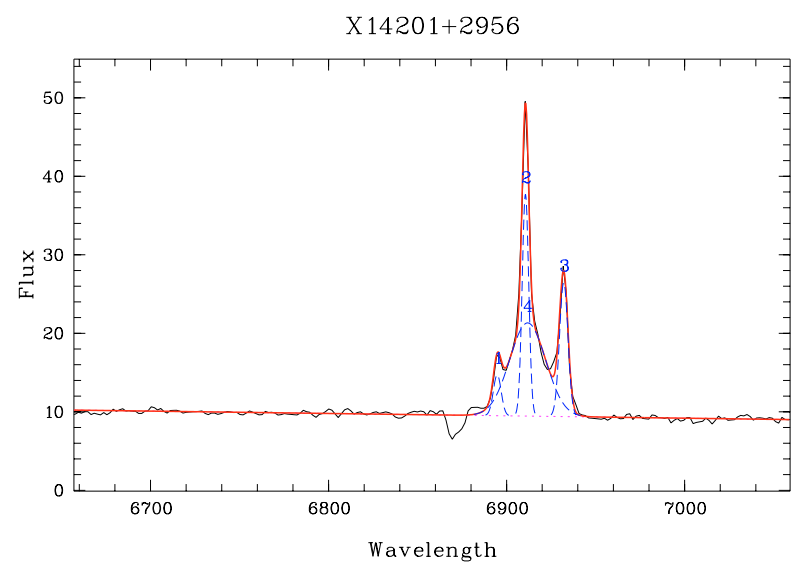

Fig. 6. IRAS 14201+2956 high dispersion spectrum (May 1998) in the $\mathrm{H}_{\alpha}$ region. The result of multi-component fiting is shown with dashedlines.

\subsection{Radio data}

We searched the publicly available radio catalogues for counterparts to our objects. IRAS $21582+1018$ was found in the NVSS survey (Condon et al. 1998) with a flux of $57.9 \mathrm{mJy}$ at 


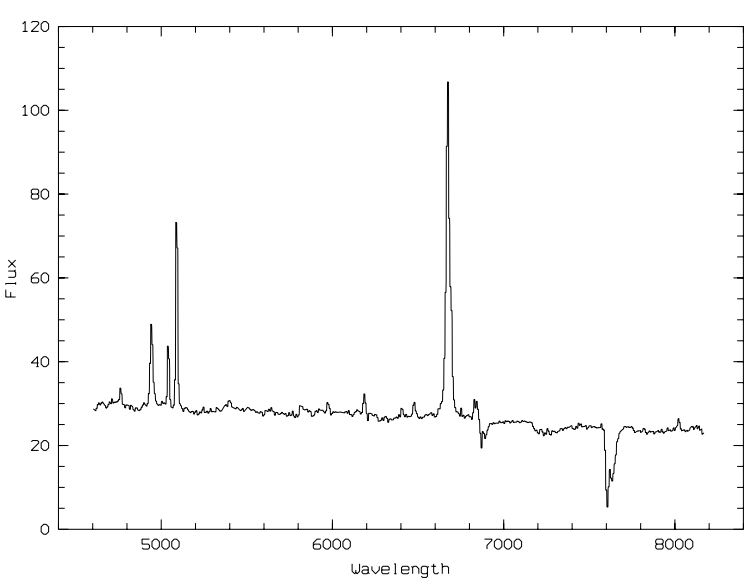

Fig. 7. Low dispersion spectrum of Mrk 359 (January 96).

$1.4 \mathrm{GHz}$, and so was the NLS1 Mrk 359, with a flux of $4.8 \mathrm{mJy}$. The other NLS1 in our list, Mrk 1388, was found in the FIRST catalogue as source $\mathrm{J} 145037.8+224403$ (the positional coincidence is better than 0 " 9 and is limited by the accuracy of the optical position) with an integrated flux of $9.93 \mathrm{mJy}$ at $20 \mathrm{~cm}$. The source IRAS $14201+2956$ is found to be associated with the source FIRST J142220.2+294255, with an integrated flux of $2.48 \mathrm{mJy}$ at $20 \mathrm{~cm}$.

\section{Discussion}

\subsection{The mid-IR properties}

The basic properties of these galaxies can be best discussed with the PHOT-S data, where all four objects have been observed. The spectrum with best signal to noise, IRAS 21582+1018 alias Mrk 520, displays several PAH lines superposed on a relatively flat continuum. The strongest line is the $7.7 \mu \mathrm{m}$, with a well separated $8.6 \mu \mathrm{m}$ line on its red shoulder. The $6.2 \mu \mathrm{m}$ line is also well detected. The sharp raise at the red extremity of the spectrum is clearly due to the presence of the $11.3 \mu \mathrm{m}$ line, partly shifted out of the observed range because of the recession velocity of this object. When comparing with the various types of objects: starbursts, AGN's and ULIRG's, observed and discussed by Lutz et al. (1998) (see for instance their Fig. 1), Mrk 520 clearly appears like a typical Starburst galaxy in the mid-IR. Rigopoulou et al. (1999) also discussed a larger sample of ULIRG's, with special emphasis on the possible effect of large extinction on the appearance of the mid-IR spectrum. The main effect of extinction is to suppress the $8.6 \mu \mathrm{m}$ line and to depress the continuum redwards of it, as shown by a comparison between M 82 and Arp 220 (their Fig. 6). No such suppression is seen here and the spectrum of Mrk 520 clearly ressembles the one of M 82 rather than Arp220. The 6.2/7.7 PAH flux ratio (0.27) is also consistent with a moderate extinction only.

Although much noisier, the spectrum of IRAS 14201+2956 (the faintest of the four sources) resembles the one of Mrk 520, with detected 7.7 and $6.2 \mu \mathrm{m}$ PAH features and a flat continuum. The $8.6 \mu \mathrm{m}$ line is not seen in the noise, while the $11.3 \mu \mathrm{m}$ one is out of range due to the higher redshift. The silicate absorption is probably strong. This spectrum is best described

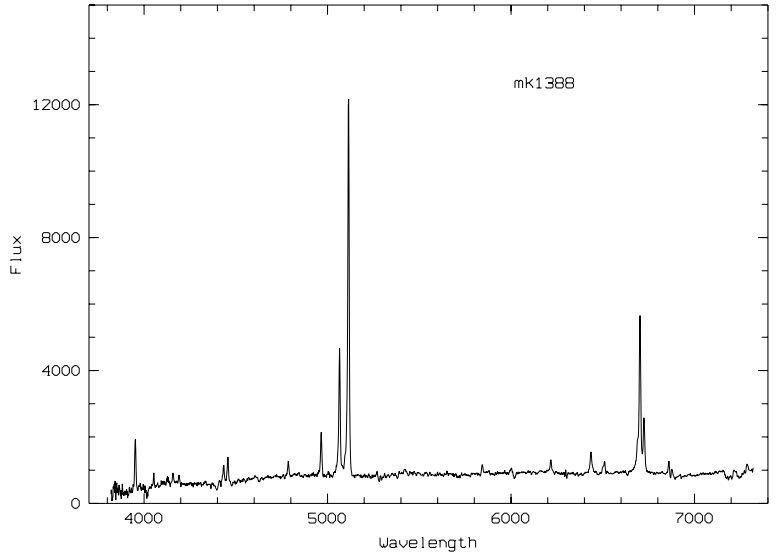

Fig. 8. Low dispersion spectrum of Mrk 1388 (June 99).

as "Starburst" when comparing to the templates of Lutz et al. (1998), but an "AGN" cannot be excluded.

By contrast, the NLS1 Mrk 359 shows only weak PAH features and a steadily raising continuum, typical of a power-law spectrum. This continuum is even detected in the usually noisy $2.4-5 \mu \mathrm{m}$ range. The $6.2 \mu \mathrm{m}$ PAH line is present with low contrast, as well as the $7.7 \mu$ one. The $11.3 \mu \mathrm{m}$ line is possibly seen at the edge of the spectrum and a weak silicate absorption may be present. This spectrum, with its higher slope and low contrast between line and continuum is reminiscent of the AGN template in Lutz et al. Although the presence of the $6.2 \mu \mathrm{m}$ line indicates also a starburst contribution, this object is clearly AGN dominated.

The last spectrum, Mrk 1388, which has the lowest signal to noise ratio, does not show any clear PAH line, but its raising continuum indicates preferentially an AGN. The slope of the continuum may be affected by a strong silicate absorption, as indicated by the sharp rise at the red end. The apparent "emission" around $9.4 \mu \mathrm{m}$, which does not correspond to any known emission line, is probably instrumental in origin.

We observed also the Pf $\alpha$ line in IRAS 14201+2956 with SWS, in the hope to reveal broad wings, signature of a Seyfert 1 . We however failed to detect the line, and derived an upper limit to its flux of $1.21 \times 10^{-20} \mathrm{~W} \mathrm{~cm}^{-2}$, with an uncertainty of about 20 percents.

Beyond the detection or not of the PAH features, the only other mid-IR information available for an identification of the nuclear source comes from the neon fine-structure lines, detected in Mrk 520, and displayed in Fig. 2. All three lines: [NeII] at $12.8 \mu \mathrm{m}$, [NeIII] at $15.5 \mu \mathrm{m}$ and [NeV] at $14.3 \mu \mathrm{m}$ are well detected with a good signal to noise ratio. The important result is that the $[\mathrm{NeV}] /[\mathrm{NeII}]$ ratio is measured at 1.2 , higher than in any other template observed by Genzel et al. (1998). Although this ratio is not corrected for reddening (as no estimate of the latter is available), its value will not change significantly for any plausible reddening value (only $10 \%$ for a screen $A_{v}$ of 50) and is a clear sign of the presence of a hard UV radiation field, i.e. an AGN. For Mrk 520, the PAH features and the fine structure lines provide therefore apparently contradictory diagnostics. 


\subsection{Comparison between IR and other wavelengths}

Putting aside for a moment the two NLS1 objects, the reason to observe the two other, "starburst", objects (IRAS 14201+2956 and IRAS 21582+1018) was their high $\mathrm{X}$-rays luminosity in the Rosat sample, rather uncommon for starburst objects. No known starburst galaxy is so X-ray luminous (Ptak et al. 1999). The mean relation between soft $\mathrm{X}$-rays and FIR luminosities derived empirically by David et al. (1992) for starburst and normal galaxies predicts an X-ray luminosity one order of magnitude lower than observed for IRAS $21582+1018$ and two orders of magnitude lower for IRAS $14201+2956$, which is far beyond the scatter of this relation. The observed X-ray luminosities, $\sim 6$ and $\sim 14 \times$ $10^{42} \mathrm{erg} \mathrm{s}^{-1}$ respectively, are, on the other hand, not uncommon among Seyfert galaxies (in our list, Mrk 1388 has in fact a lower X-ray luminosity than these two objects!).

The low dispersion optical spectra do not show conspicuous signs of a Seyfert nature for IRAS 14201+2956 and IRAS $21582+1018$ (this was the starting problem when they were detected in the Rosat survey). In the course of this work, it appeared that IRAS 14201+2956 had been detected in the Einstein Medium Sensitivity Survey (Gioia et al. 1990), and quoted as an AGN without further precision. The spectroscopic follow-up by Stocke et al. (1991) is based on a blue spectrum only (without $\mathrm{H}_{\alpha}$ ), and does not give a more precise classification: as judged from our own spectra at similar resolution, this object was therefore among their 8 percent of objects where the classification was based on $[\mathrm{OII}] /[\mathrm{OIII}]$ ratios only, and thus requiring additional observations. No further spectrum was taken by Moran et al. (1996) in their Rosat follow-up, so it is not clear where their S1 classification is coming from. Only the higher dispersion spectra presented here show unambiguously the presence of a broad component around $\mathrm{H}_{\alpha}$ (Fig. 4): this object can therefore be classified now as a Sey 1.9, as, indeed, no similar broad component is detected around $\mathrm{H}_{\beta}$ (although the detection limit might be improved). The excitation measured by the $[\mathrm{OIII}] / \mathrm{H}_{\beta}$ ratio is around 1 , therefore a priori excluding a classical Seyfert 2 galaxy.

For IRAS $21582+1018$, no previous spectra were available and our first classification spectrum did not reveal broad components, but an excitation close to the border line between starburst and Seyfert galaxies. A better spectrum obtained in 1996 (Fig. 4) confirms those line ratios, placing the object close to the border line but on the Seyfert side on the diagnostic diagrams presented by Veilleux et al. (1999) for Iras galaxies. The decisive ratio is the [OIII] $5007 \AA / \mathrm{H}_{\beta}$ one with a value of 5 , while no broad $\mathrm{H}_{\alpha}$ component is seen, therefore pointing towards a Seyfert 2. The presence of the broad $\mathrm{H}_{\alpha}$ component is only revealed in our high-dispersion spectrum by a multicomponent analysis: three narrow components with similar width $\left(\mathrm{H}_{\alpha}\right.$ and the two [NII] lines) and one $\mathrm{H}_{\alpha}$ component with greater width were adjusted to reproduce the observed complex. The result of the fit is shown in Fig. 5 with dashed lines: the broad $\mathrm{H}_{\alpha}$ component has an amplitude 7 times smaller than the narrow component and a FWHM of $1800 \mathrm{~km} \mathrm{~s}^{-1}$. The observed width of the narrow components is $310 \mathrm{~km} \mathrm{~s}^{-1}$, while the instrumental profile is $180 \mathrm{~km} \mathrm{~s}^{-1} \mathrm{FWHM}$. The total intensity of the broad $\mathrm{H}_{\alpha}$ component is about equal to that of the narrow component. This object is thus also classified here as a Sey 1.9, in accordance with the classification proposed by Moran et al. (1996) from a spectrum at $\sim 5 \AA$ resolution. From the ratio of the narrow components of $\mathrm{H}_{\alpha} / \mathrm{H}_{\beta}$, we derive a reddening of $A_{V}=1.4$ mag under the assumption of case $\mathrm{B}$ recombination.

A similar multi-component analysis made for IRAS 14201+2956 (Fig. 5) gives an intensity for the broad $\mathrm{H}_{\alpha}$ component of 2.2 times the intensity of the narrow component, and a FWHM of only $1030 \mathrm{~km} \mathrm{~s}^{-1}\left(220 \mathrm{~km} \mathrm{~s}^{-1}\right.$ for the narrow components). The $\mathrm{H}_{\alpha} / \mathrm{H}_{\beta}$ ratio gives here a reddening essentially zero, with however a significant uncertainty. The optical classification of both objects as Sey 1.9 is thus now in agreement with the detection of a strong soft X-rays component.

For the NLS1 objects, our spectra do not reveal any significant new feature compared to previous classification. Mrk 359 was observed in details by Veilleux (1991), who noted its small line widthes and the apparent absence of reddening in the narrow line region. For Mrk 1388, the excitation is extremely high, a feature already noticed by Osterbrock (1985), and which is more appropriate for Seyfert 2 galaxies, than for Seyfert 1s. No substantial reddening is indicated here for those objects either.

While the X-ray and optical observations are now in agreement with an AGN classification for IRAS 14201+2956 and IRAS $21582+1018$, the contradiction of the broad IR features with this interpretation remains. The PAH features detected by ISO-PHOT characterise starburst galaxies. The broad SED in the far IR, from 10 to $100 \mu \mathrm{m}$, is also typical of starburst galaxies and does not satisfy the various criteria defined to select AGN in IRAS data (de Grijp et al. 1985; Désert \& Dennefeld 1988).

On the contrary, both Mrk 359 and Mrk 1388, the NLS1 galaxies, stand out with a mid-IR excess around $25 \mu \mathrm{m}$, typical of warm dust heated by an AGN, the excess being particularly strong for Mrk 1388.

If we use the radio data, to compute the standard IR/radio parameter $q$ discussed by Condon at al. (1995), we find a value of 2.52 for IRAS 14201+2956, again typical of starburst galaxies and showing that the AGN, if present, is not dominating the IR emission and/or that the object is radio-weak. For Mrk 359, the $q$ parameter has the value 2.51, which is not typical of a strong AGN, and thus points probably towards a radio-weak nature. For Mrk 1388, on the contrary, this ratio is much lower (1.54), indicating the predominance of the AGN. The case of IRAS $21582+1018$ is intermediate, with $q=2.01$, reflecting the complexity of this source and the mix of starburst and AGN.

Finally, the observed infrared luminosities for the two Sey 1.9 s, IRAS $14201+2956$ and IRAS $21582+1018$, are very high if we assume that the objects have an $L_{\mathrm{IR}} / L_{\mathrm{X}}$ ratio typical for AGN or quasars with $L_{\mathrm{X}}<10^{45} \mathrm{erg} \mathrm{s}^{-1}(\simeq 4.5$; Elvis et al. 1994): extrapolating the Rosat fluxes and correcting for absorption provides a $0.5-10 \mathrm{keV}$ luminosity of 4 and $2 \times 10^{44} \mathrm{erg} \mathrm{s}^{-1}$, which translates into an $L_{\mathrm{IR}} / L_{\mathrm{X}}$ ratio of 16 and 45 respectively. The bulk of the IR emission has therefore to be attributed to another source than the AGN. The fact that IRAS 14201+2956 is 
located close to another galaxy (included in the Iras lobe) could mean that this second object is also contributing to the far IR emission, but we can rule out a significant contribution from it because its optical spectrum is not the one of a typical Iras starburst galaxy (no emission lines present).

The discrepancy between X-ray and IR signatures is therefore best resolved if the two emissions have a different origin. The X-rays come from a central AGN, whose contribution to the IR emission is negligible. The bulk of the IR radiation is produced by an intense star formation episode, occurring on spatial scales of the order of or larger than the NLR, and not directly linked to the AGN. The limited angular resolution of our ground-based spectra ( 1 " 2 per pixel, with an average seeing of $2^{\prime \prime}$ ) does not allow us to disantangle spectroscopically the AGN from the starburst region: we can only say that this star forming region must be contained within a "central" region of about $3 \mathrm{kpc}$ diameter for IRAS $14201+2956$ and $2 \mathrm{kpc}$ for IRAS $21582+1018$. This is completely consistent with what is known for other, starbursting, IRAS galaxies. However the fact that the neon line ratios clearly indicate an AGN in IRAS $21582+1018$ means that the dusty starburst is not fully obscuring the view to the central region. The other question is then to understand why the optical spectrum hardly reveals the broadline region, while the $\mathrm{X}$-rays are coming out: the measured optical extinction of $A_{V} \sim 1.4$ is largely unsufficient to hide it. The column density derived from the X-ray spectral analysis provides only $A_{V}=1$, barely consistent with the value derived from the optical: there is therefore no room in the X-rays for additional extinction towards the center. The measured optical extinction probably refers then to an outer region only (starburst/NLR), possibly linked to a dusty, warm absorber, as known in other cases (e.g. Reynolds et al. 1997). This is another argument for a distinct origin of the IR and Xray emission. For IRAS $14201+2956$, both the optical and the $\mathrm{X}$-ray indicate an absorption close to zero, but the broad $\mathrm{H}_{\alpha}$ component is also better detected.

We can also use an isotropic indicator of the AGN's intrinsic brightness, like the [OIII] luminosity, corrected for extinction, and compare it to the $1-10 \mathrm{keV}$ luminosity (derived by extrapolation from the Rosat data) to get an estimate of the absorption affecting the X-rays flux (Maiolino et al. 1998; Bassani et al. 1999). Using our 1996 spectrum for IRAS $21582+1018$ (obtained in photometric conditions), we derive a $L_{\mathrm{X}} / L_{\mathrm{OIII}}$ ratio of 1.3 only, on the very low side of the bulk of Seyfert 1 galaxies (Maiolino et al. 1998, their Fig. 6), thus leaving room for at least a moderate absorption, although a compton-thick source is difficult to justify with the available data (as a comparison, the same ratio derived for Mrk 1388 is about 10, a value typical for Sey 1 galaxies). For IRAS $14201+2956$, this ratio is much higher $(\sim 200)$ : even if it is affected by a large uncertainty (a factor of 2) on the [OIII] luminosity, this object is probably not heavily absorbed. In both cases however, one has to remember that the X-rays fluxes used in the calculation are extrapolated from soft data, and could therefore be overevaluated due to the possible presence of a strong soft X-rays excess.

We could therefore propose that these two objects are better described as weak AGNs rather than highly obscured objects.
From their optical properties, they refer mainly to the Sey 1.9 or 1.8 classes, where the reason for weak broad lines is still not understood. As they have a large soft X-ray component, they could be closer than suspected to NLS1s, where the extinction is small also. It will therefore be important to get hard X-ray observations for all these objects, to determine the relative importance of the soft X-ray component (excess or not), to check the existence of a dusty, warm absorber and to assess the real strength of the central AGN and the obscuration in front of it. Confirming the possible variability is another element to clarify their nature. They may in the end be local examples of the absorbed type- 2 objects searched for at much higher redshift.

\section{Conclusion}

A combination of optical, IR and X-ray observations has helped to clarify the apparent discrepancies in the properties of the two galaxies IRAS 14201+2956 and IRAS 21582+1018. The high soft X-ray emission detected by Rosat, which is difficult to explain by a starburst, is linked to a central AGN whose presence is detected in the optical only by high resolution spectroscopy revealing a weak, broad component in $\mathrm{H}_{\alpha}$. The emission and spectral energy distribution in the IR, on the other hand, is clearly dominated, even in the ISO mid-IR range, by a circumnuclear starburst revealed by the PAH features. The discrepancy between the X-ray and IR properties is therefore best explained by a different origin of the main emissions. In the case of IRAS $21582+1018$ however, high resolution spectroscopic observations with ISO have also revealed highexcitation lines clearly associated with the AGN and its NLR, but not dominating the energy balance in the IR. The extinction measured from the X-ray or the optical is insufficient to explain the lack of strong, broad emission lines and pertains essentially to the starburst/NLR only. IRAS $21582+1018$ and to a lesser extent IRAS 14201+2956 are therefore best described by a central, perhaps weak, AGN, with a BLR partly obscured by a structured absorber, and an absorbed NLR, mixed with a region of intense star formation of perhaps larger extension. These examples show that the detection of AGN spectral line characteristics in the optical or IR are not enough to establish the main source of energy in the IR and that mixed cases may be frequent. Many of them are probably found in the B92 and B98 samples, as the selection there was made both by X-ray and IR emission. The only established common property between those objects and the NLS1 galaxies also found in large number in these samples (of which two examples, Mrk 359 and Mrk 1388, were studied here) seems to be the strong soft X-ray emission. Whether or not both type of objects have more common properties, and are for instance both characterized by a weak central AGN remains to be established: only hard X-ray observations will be able to measure the strength of the central engine and determine the surrounding absorption.

Acknowledgements. This research has made use of the LEDA Extragalactic Database, which is operated at Observatoire de Lyon (France), and of data obtained through the High Energy Astrophysics Science Archive Research Center Online Service, provided by the NASA/Goddard Space Flight Center. We thank the anonymous referee for helpful comments. 


\section{References}

Bassani, L., Dadina, M., Maiolino, R., et al. 1999, ApJS, 121, 473 Boller, T., Meurs, E. J. A., Brinkmann, W., et al. 1992, A\&A, 261, 57 Boller, T., Dennefeld, M., Brinkmann, W., et al. 1993, in First light in the Universe: Stars or QSOs?, ed. B. Rocca-Volmerange, B. Guiderdoni, M. Dennefeld, \& J. Tran Thanh Van (Éditions Frontières), 339

Boller, Th., Bertoldi, F., Dennefeld, M., \& Voges, W. 1998, A\&AS, 129,87

Condon, J. J., Andreson, E., \& Broderick, J. J. 1995, AJ, 109, 2318

Condon, J. J., Cotton, W. D., Greisen, E. W., et al. 1998, AJ, 115, 1693

David, L. P., Jones, C., \& Forman, W. 1992, ApJ, 388, 82

Désert, F. X. D., \& Dennefeld, M. 1988, A\&A, 206, 227

Dickey, J. M., \& Lockman, F. J. 1990, ARA\&A, 28, 215

Elvis, M., Wilkes, B., McDowell, J. C., et al. 1994, ApJS, 95, 1

Genzel, R., Lutz, D., Sturm, E., et al. 1998, ApJ, 498, 579

de Grijp, M. H. K., Miley, G. K., Lub, J., \& de Jong, T. 1985, Nature, 314,240

Gioia, I. M., Maccacaro, T., Schild, R. E., et al. 1990, ApJS, 72, 567

Heckman, T. M., Armus, L., \& Miley, G. K. 1987, AJ, 93, 276

Kessler, M. F., Steinz, J. A., Anderegg, M. E., et al. 1996, A\&A, 315, L27

Lemke, D., Klaas, U., Abolins, J., et al. 1996, A\&A, 315, L64
Lutz, D., Spoon, H. W. W., Rigopoulou, D., Moorwood, A. F. M., \& Genzel, R. 1998, ApJ, 505, L103

Maiolino, R., Salvati, M., Bassani, L., et al. 1998, A\&A, 338, 781

Markarian, B. E., \& Lipovetskii, V. A. 1971, Astrophys., 7, 299

Markarian, B. E., \& Lipovetskii, V. A. 1974, Astrophys., 9, 283

Markarian, B. E., Lipovetskii, V. A., \& Stepanyan, D. A. 1980, Astrophys., 15, 363

Moran, E. C., Halpern, J. P., \& Helfand, D. J. 1994, ApJ, 433, L65

Moran, E. C., Halpern, J. P., \& Helfand, D. J. 1996, ApJS, 106, 341

Osterbrock, D. E. 1985, PASP, 97, 24

Ptak, A., Serlemitsos, R., Yaqoob, T., \& Mushotzky, R. 1999, ApJS, 120,179

Reynolds, C. S., Ward, M. J., Fabian, A. C., \& Celotti, A. 1997, MNRAS, 291, 403

Rigopoulou, D., Spoon, H. W. W., Genzel, R., et al. 1999, AJ, 118, 2625

Sanders, D. B., \& Mirabel, I. F. 1996, ARA\&A, 34, 749

Stocke, J. T., Morris, S. L., Gioia, I. M., et al. 1991, ApJS, 76, 813

Tran, H. D., Osterbrock, D. E., \& Martel, A. 1992, AJ, 104, 2072

Veilleux, S. 1991, ApJ, 368, 158

Veilleux, S., Kim, D. C., Sanders, D. B., Mazzarella, J. M., \& Soifer, B. T. 1995, ApJS, 98, 171

Veilleux, S., Kim, D. C., \& Sanders, D. B. 1999, ApJ, 522, 113 